\title{
Operation of a Sunpower M87 Cryocooler in a Magnetic Field
}

\author{
S.R. Breon, K.A. Shirey, I.S. Banks, B.A. Warner, R.F. Boyle, and S. Mustafi \\ NASA Goddard Space Flight Center \\ Greenbelt, ML, USA 20771
}

\begin{abstract}
The Alpha Magnetic Sp:ctrometer-02 (AMS-02) is an experiment that will be flown as an attached payload on the International Space Station to detect dark matter and antimatter. It uses large superconducting magnets cooled with superfluid helium to bend the path of cosmic particles through a series of detectors, which then measure the mass, speed, charge, and direction of the particles. Four Sunpower M87N Stirling-cycle cryocoolers are used to extend the mission life by cooling the outer vapor-cooled shield of the dewar. The main magnet coils are separated by a distance of approximately $1 \mathrm{~m}$ and the coolers are located approximately $1.5 \mathrm{~m}$ from the center line of the magnet, where the field is as high as 925 gauss perpendicular to the cryocooler axis and 400 gauss along the cryocooler axis. Interactions between the applied magnetic field and the linear motor may result in additional forces and torques on the compressor piston. Motion of the compressor and displacer pistons through the magnetic field spatial gradients will generate eddy currents. Additional eddy currents are created during magnet charge, discharge, and quench by the time-var ing magnetic field. The results of tests to determine the magnitude of the forces, torques, and heating effects, as well as the need for additional magnetic shielding, are presented.
\end{abstract}

\section{INTRODUCTION}

The Sunpower M87N cryocoolers that are used on AMS-02' are exposed to substantial steady-state magnetic field, that could interfere with the thermal performance of the cooler or degrade the cryocooler's lifetime. Either of these effects would shorten the mission duration, since the purpose of the cryocoolers is to reduce the parasitic heat load and extend the lifetime of the AMS-02 superfluid helium dewar. It is conceivable that the coolers would not operate at all, or could be damaged by edcly current forces during a magnet quench.

AMS-02 is the first space-flight mission that has been faced with these issues. Studies of the Astromag project ${ }^{2}$ considered the use of cryocoolers for a free-flyer version, but the technology development effort to examine this question was never funded. The XRS instrument $^{3}$, which was lost due to a launch failure, is being rebuilt for launch on Astro-E2. XRS does have a superconducting magnet and the XRS2 version will use a cryocooler to extend the life of the superfluid heliunı dewar,. Compared to AMS-02, however, the XRS2 magnet is much more modest in size and field, and the magnet is heavily shielded. The field at the XRS2 cryocooler will be $<10$ gau $s$. 
For ground applications, Gifford-McMahon (GM) cryocoolers have been used to cool Magnetic Resonance Imagin (MRI) magnets ${ }^{4,5,6}$. Reports on these applications have focused more on how effectively the cryocooler cools the magnet and whether or not the vibrations from the cryocooler disturb the MRI magnet or system. A small performance loss of the cryocooler would likely go unnoticed. Furthermore, in MRI applications the cryocooler can be located in a relatively low magnetic field.

\section{EXTERNAL MAGNETIC FIELD}

AMS-02 uses four cryoc oolers: two are mounted on the upper support ring and two on the lower support ring, as showr in Fig. 1. The axes of the cryocoolers are aligned radially to the rings. Each cryocooler experiences different field strengths and field gradients depending on its direction. Due to symmetry the fields and gradients at the cryocoolers on the upper ring are a mirror image of each other. The same is true on the lower ring.

The magnet is being designed and built by Space Cryomagnetics, Ltd (SCL). Using their model of the AMS-02 magnet, SCL generated values of the magnetic field in all three directions at 40 grid points specified for each cryocooler. The values of the fields, given in AMS-02 coordinates, were translated to cryocooler coordinates. The grid points are shown in Fig. 2 . The values of the field for one of the cryocoolers on the upper ring are given in Table $1 . B_{x}$ is the field parallel to the cylindrical axis of the cryocooler. $B_{y}$ and $B_{z}$ are perpendicular to the cryocooler axis. $B_{y z}$ is the resultant of $B_{y}$ and $B_{z}$ and shows the magnitude of the field normal to the cryocooler axis. Table 2 shows the maximum field gradients of the fields along the cryocooler axis and normal to the axis.

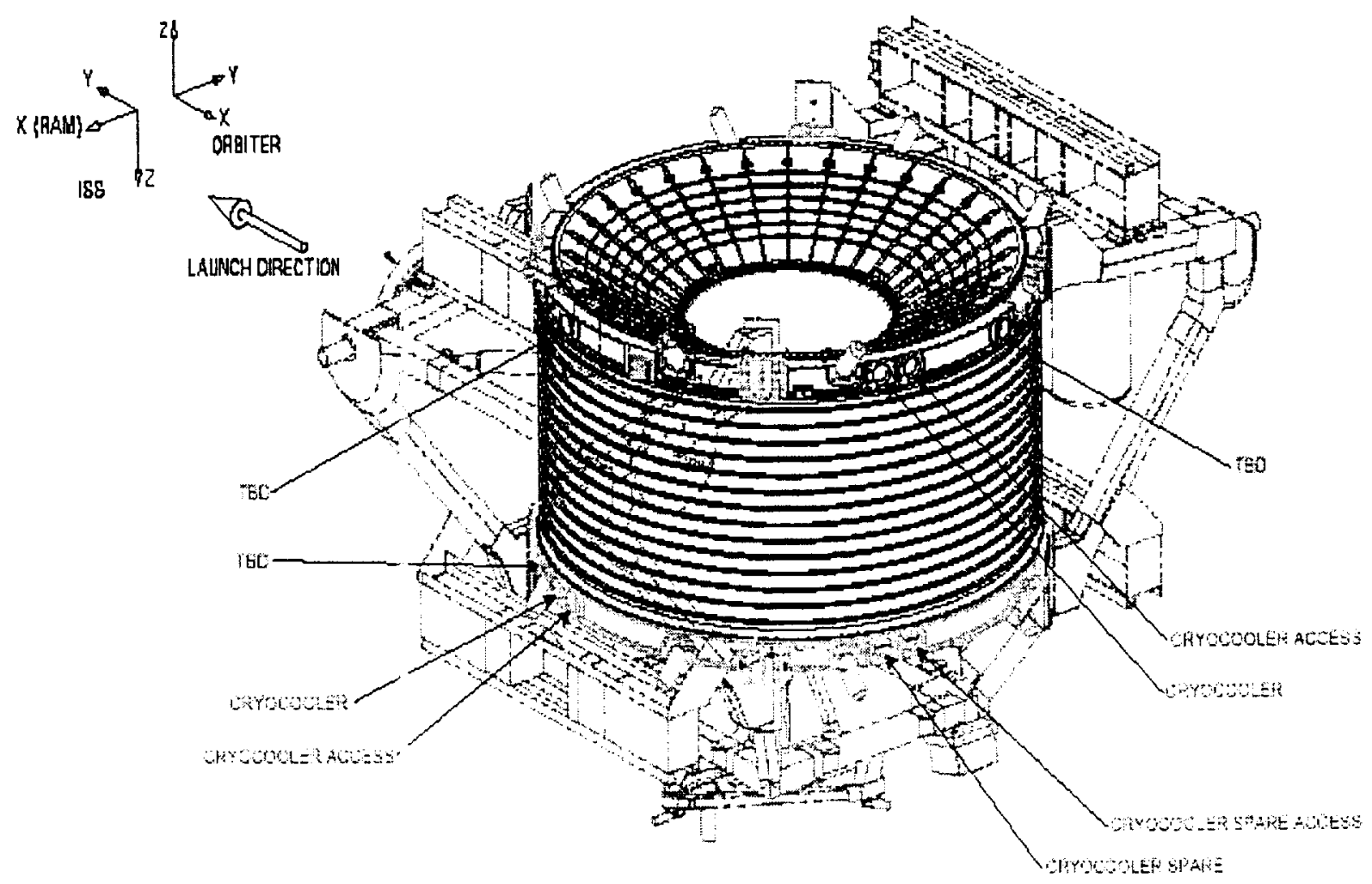

Figure 1: Cryocooler port locations on AMS-02 vacuum case. 


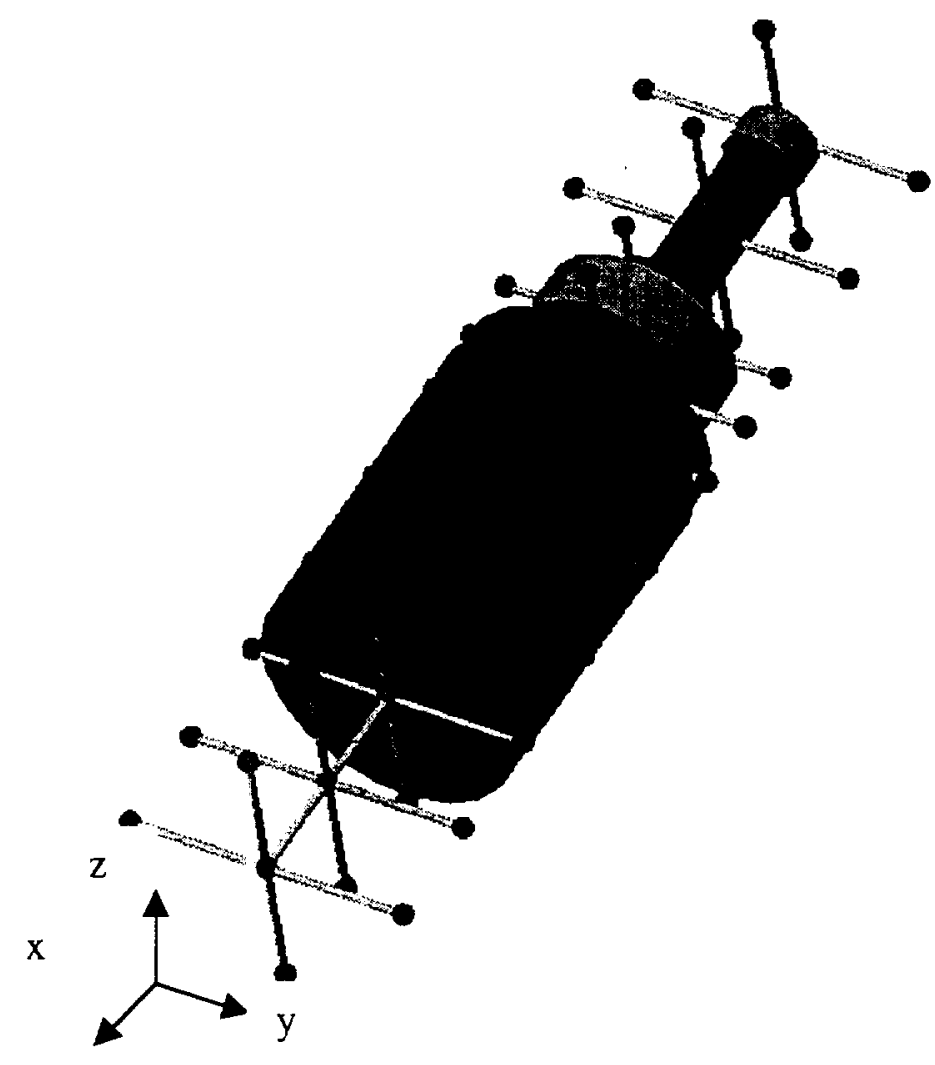

Figure 2: Cryocooler based co-ordinate system and node matrix.

Tible 1: Magnetic field at select locations

\begin{tabular}{|l|c|c|c|c|c|}
\hline \multicolumn{1}{|c|}{ Node Location } & $\begin{array}{c}\mathrm{B}_{\mathrm{x}} \\
\text { (gauss) }\end{array}$ & $\begin{array}{c}\mathrm{B}_{\mathrm{y}} \\
\text { (gauss) }\end{array}$ & $\begin{array}{c}\mathrm{B}_{\mathrm{z}} \\
\text { (gauss) }\end{array}$ & $\begin{array}{c}\mathrm{B}_{\mathrm{xy}} \\
\text { (gauss) }\end{array}$ & $\begin{array}{c}|\mathrm{B}| \\
\text { (gauss) }\end{array}$ \\
\hline Cold tip center & -47 & 504 & -648 & 506 & 822 \\
\hline Cold tip $+\mathrm{Y}$ & -93 & 457 & -698 & 466 & 839 \\
\hline Cold tip $-\mathrm{Y}$ & 4 & 543 & -591 & 543 & 802 \\
\hline Cold tip $+Z$ & 10 & 452 & -568 & 452 & 726 \\
\hline Cold tip $-Z$ & -121 & 559 & -732 & 572 & 929 \\
\hline Motor center & -129 & 348 & -432 & 371 & 569 \\
\hline Motor $+Y$ & -161 & 317 & -460 & 356 & 581 \\
\hline Motor $-Y$ & -93 & 374 & -399 & 385 & 555 \\
\hline Motor $+Z$ & -84 & 318 & -394 & 329 & 514 \\
\hline Motor $-Z$ & -181 & 379 & -468 & 420 & 629 \\
\hline Balancer center & -138 & 271 & -324 & 305 & 445 \\
\hline Balancer $+Y$ & -163 & 248 & -344 & 297 & 454 \\
\hline Balancer $-Y$ & -111 & 291 & -302 & 312 & 434 \\
\hline Balancer $+Z$ & -104 & 251 & -302 & 271 & 406 \\
\hline Balancer $-Z$ & -177 & 293 & -345 & 342 & 486 \\
\hline
\end{tabular}

During most of the time on-orbit, the magnetic field applied by the AMS02 superconducting magnet will be constant. The magnet is first charged once AMS-02 is installed on the International Space Station ind a persistent switch is closed. It is not expected that the magnet will be discharged and recharged frequently, if ever, on orbit. In the event of a quench, the magnet's protection circuit will discharge the magnet rapidly over a period of 4 seconds. The magnet quench profile is shown in Fig. 3. The maximum magnetic field rate of change is TBD. 


\begin{tabular}{|l|l|l|l|l|l|l|}
\hline \multicolumn{1}{|c|}{ Node Location } & & & & & & \\
\hline Cold tip center & & & & & & \\
\hline Cold tip $+Y$ & & & & & & \\
\hline Cold tip $-Y$ & & & & & \\
\hline Cold tip $+Z$ & & & & & & \\
\hline Cold tip $-Z$ & & & & & & \\
\hline Motor center & & & & & & \\
\hline Motor $+Y$ & & & & & & \\
\hline Motor $-Y$ & & & & & & \\
\hline Motor $+Z$ & & & & & & \\
\hline Motor $-Z$ & & & & & & \\
\hline Balancer center & & & & & & \\
\hline Balancer $+Y$ & & & & & & \\
\hline Balancer $-Y$ & & & & & & \\
\hline Balancer $+Z$ & & & & & & \\
\hline Balancer $-Z$ & & & & & \\
\hline
\end{tabular}

\section{MAGNETIC FIELD/CRYOCOOLER INTERACTIONS}

The focus of our investigation is the effect that the applied magnetic field has on the cryocoolers, which have a fixed coil, moving magnet ac linear motor. Although the cryocoolers will perturb the external field locally, they are far removed from the central bore of the magnet system and the disturbance at the location of the detectors is expected to be negligible. The magnet developer may inclide the cryocoolers in a future iteration of the AMS-02 magnetic field model.

When the cryocoolers are turned off and the field is at steady-state, the external field will interact with any magnetic materials on the cryocooler. Outside of the motor, the only significant magnetic material is a steel ring that is part of the counterbalance. For reasons that are explained later in this paper, we are considering replacing this ring with a non-magnetic stainless steel ring. The motor itself is designed to have no net dipole moment, so any induced magnetic forces on the cryocooler will be due to non-ideal characteristics of the motor such as mechanical tolerances or varation in the magnetization of the material. If the field were strong enough, it could alter the magnetization of the cryocooler motor.

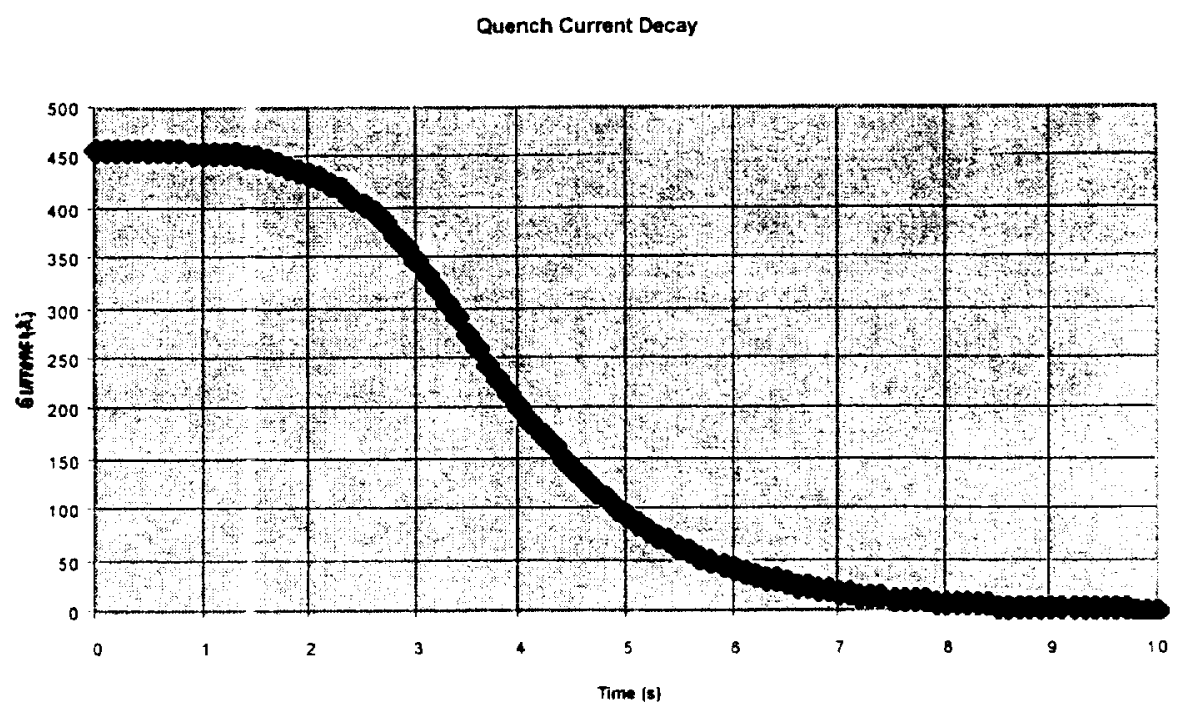

Figure 3: Currt nt quench profile of the AMS-02 superconducting magnet. 
For most of the time on-1)rbit the cryocoolers will be operating in a non-uniform steady-state magnetic field. Forces and torques on the moving magnet could cause misalignment of the compressor piston and caus vibration and rubbing, which could shorten the lifetime of the cryocooler. If the external magnetic field saturates the motor laminates, it will reduce the motor efficiency and degrade the thermal performance. In addition to effects on the magnetic materials, the external magnetic field will generate eddy currents in all moving metallic pieces such as the displacer and the compressor pistons. These eddy currents will generate heat that will reduce the cryocooler thermal performance. Eddy current forces will tend to inhibit the motion of the pistons, thus further degrading thermal performance.

When the magnet is being charged or discharged, or if it quenches, eddy currents will be generated in all metal components in the cryocooler. Since these events will be rare, any degradation in thermal performance during a transient will not be of concern to the project. The main concern is that the transients not cause damage to the cryocoolers.

\section{TEST RESULTS}

We tested two engineering model cryocoolers in a magnetic field at a cyclotron facility located at the Massachusetts Institute of Technology (see Fig. 4.) At the center of the cyclotron, the field is very uniform. Near the edges, however, there is a substantial gradient in the radial direction. At the midplane between the two poles, the cyclotron creates a vertical magnetic field. After mapping out the magnetic field we chose to place the cryocooler in a horizontal position in the fringing field near the edge of the cyclotron. The magnetic field at the motor is considered to be the most important, so the vertical field at the center of the motor was chosen to be the standard for the tests: the cold tip was in a larger field and the balancer was in a smaller field during the test compared to the flight configuration. Although the cyclotron does generate a radial field (axial to the cryocooler), above the midplane the field is radially outward and below the midplane it is radially inward, so it does not mimic the AMS-02 field.

The maximum expected field at the cryocooler motor is 610 gauss perpendicular to the cryocooler axis. We were ahle to operate both cryocoolers at full field with no degradation in performance. Both cryocoolers continued to operate up to 1,200 gauss, or twice the maximum expected field, with a small degradation in performance. The cryocoolers were cycled on and off when exposed to a 1,200 gausis field without incident.

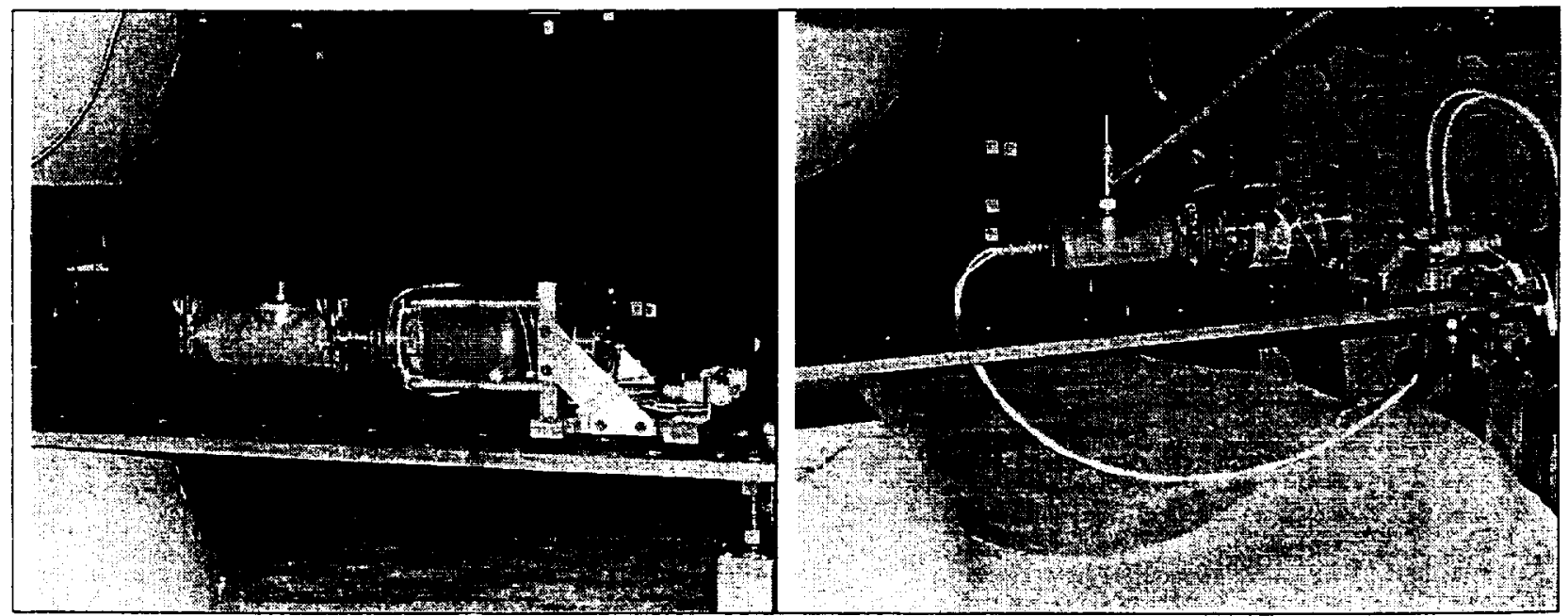

Figure 4: Engineering Model Cryocoolers 1 (left) and 2 (right) mounted in the cyclotron facility 


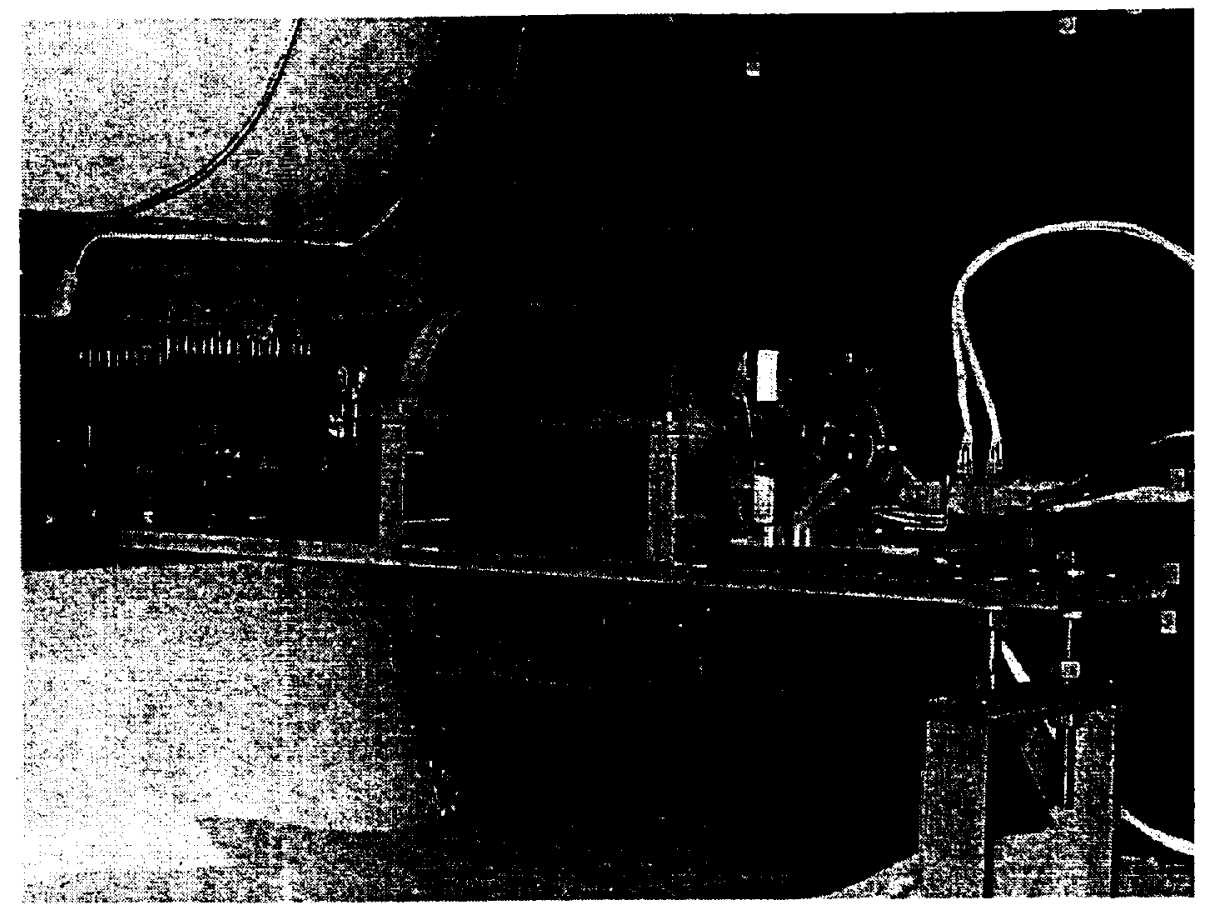

Figure 5: Cryocooler in a magnetic shield

The cryocoolers were tested with magnetic shields to determine if shielding would reduce or eliminate the performance degradation, as shown in Fig. 5. Two shields were tested. Both were cylindrical iron shields exten ting from the cold tip to the aft end of the compressor. One shield had an outer diameter of $135 \mathrm{~mm}$ and a thickness of $4.6 \mathrm{~mm}$. The other shield was $152 \mathrm{~mm}$ in diameter and $12.7 \mathrm{~mm}$ thick. Both shields were $279 \mathrm{~mm}$ long. Although the shields did screen out some of the external field they had no effect on the performance degradation.

To explore the performance degradation more carefully, a systematic set of load curves was taken for one of the engineer ng model cryocoolers at three input powers and three heat loads as a function of increasing magrietic field. The results, shown in Fig. 6, are corrected for drift in the heat sink temperature around a nominal $25^{\circ} \mathrm{C}$. The minimum and maximum input power at which the cryocoolers can b: operated is $60 \mathrm{~W}$ and $150 \mathrm{~W}$, respectively. Their nominal input power is $100 \mathrm{~W}$. The effect is most pronounced at low input power and high heat load: at $60 \mathrm{~W}$ input power and $6 \mathrm{~W}$ heat load, there is a $4 \%$ loss in cooling power at $\sim 900$ gauss, or 1.5 times the maximum expected field. At $100 \mathrm{~W}$ input power and $6 \mathrm{~W}$ heat load, the cooling loss at $\sim 900$ gauss is less than $1 \%$.

One possible explanatior for the performance degradation was that the thermometers used to measure cold tip temperatur: were affected by the magnetic field. This would not have been expected because the Cernox thermometers we used are supposed to be insensitive to magnetic fields. However, to verify that the problem was not associated with the thermometry, we rotated the cryocoolers to place the cold tip in a lower magnetic field. In doing so, we discovered to our surprise that the performance degradation increased. We then realized that the balancer was now in a higher field, and the interaction between the magnetic steel in the balancer and the external magnetic field was hurting the cryocooler performance.

To test this hypothesis we mounted the cooler on its flexible mount and took load curves with the balancer attached and with the balancer removed. We also rigidly mounted the cryocooler with the balancer attached. The results of these tests are shown in Fig. 7. As expected, the cryocooler on a flexible mount with the halancer attached shows a larger rise in temperature as well as sensitivity at lower fields than the other two cases. When the cryocooler is mounted on a flexible mount and the balancer is "unbalanced," a greater unbalance results in a warmer cold-tip temperature. One way of explaining this is that some of the energy going into the cryocooler is now being dissipated in viscous damping at the mount rather than in useful work on the gas.

This explains both the higher operating temperature when the cryocooler has no balancer at all as well as the cold tip temperature increase for a flexibly mounted cryocooler 
Heat Load: 0 W

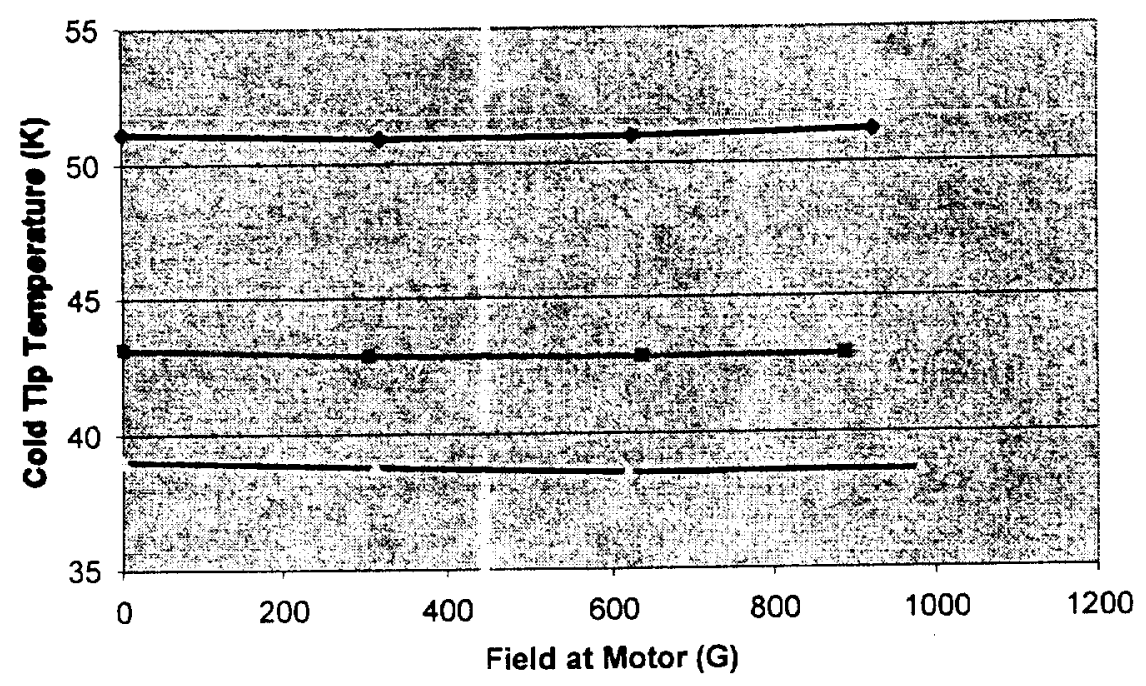

Heat Load: 3 w

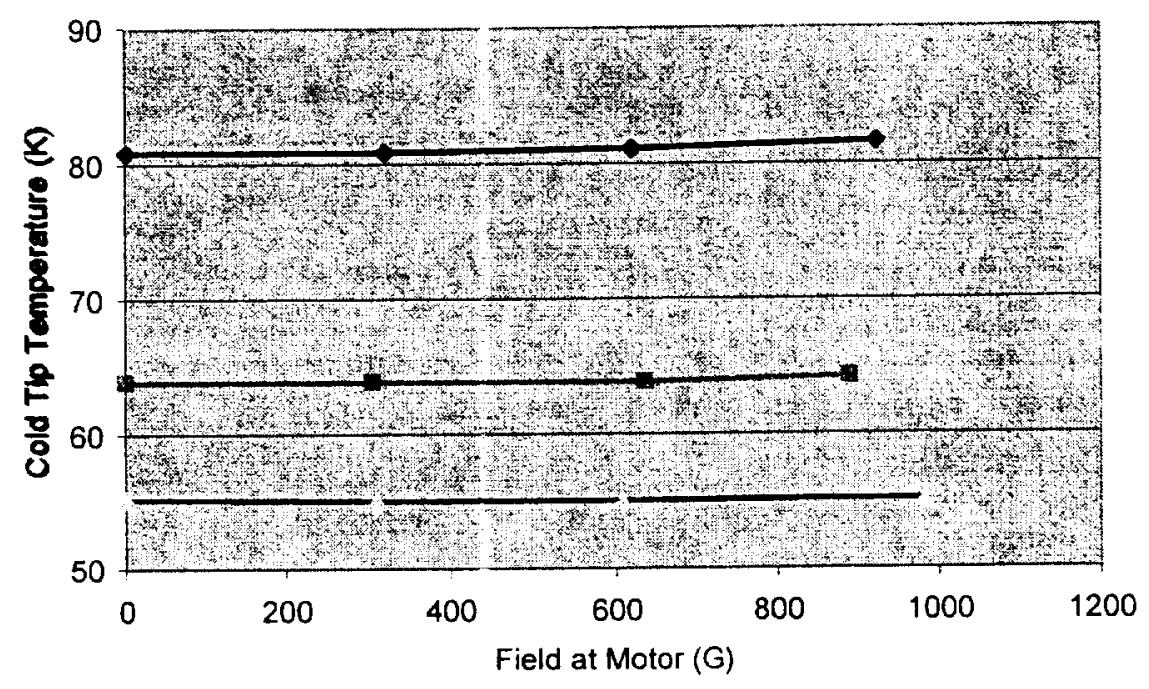

Heat Load: 6 W

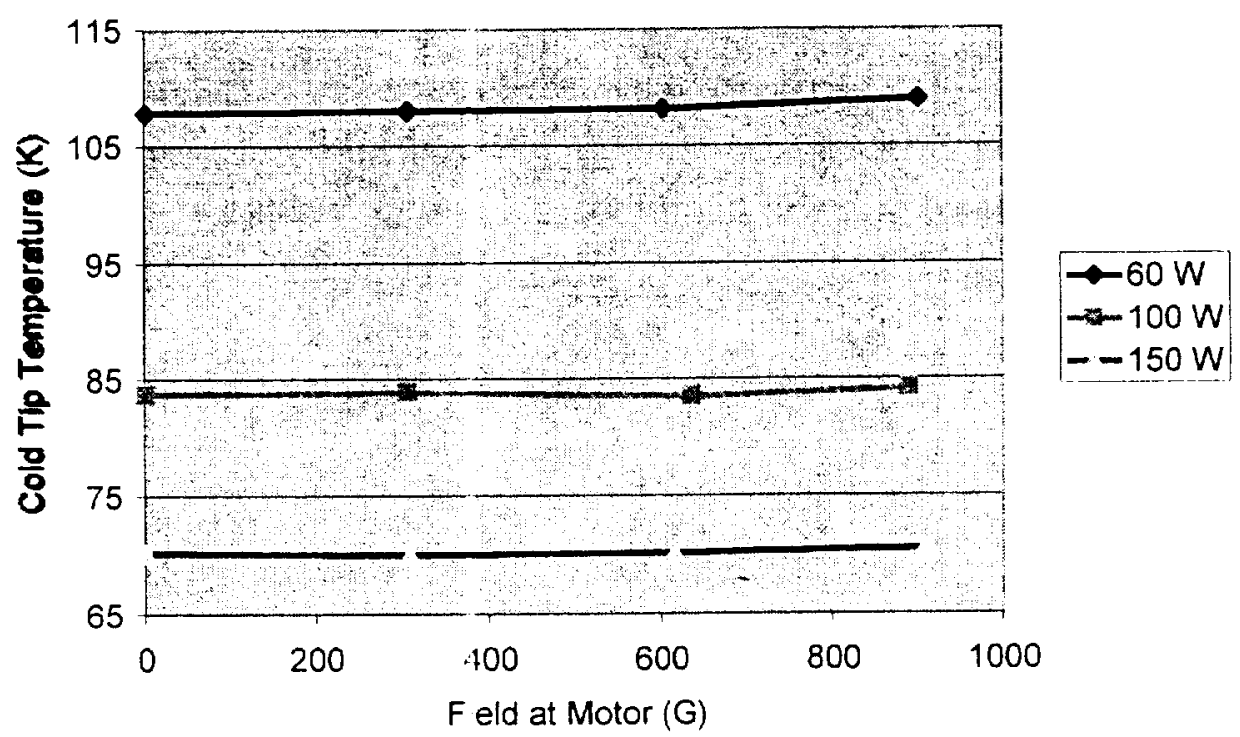

Figure (": Cryocooler load curves as a function of magnetic field 


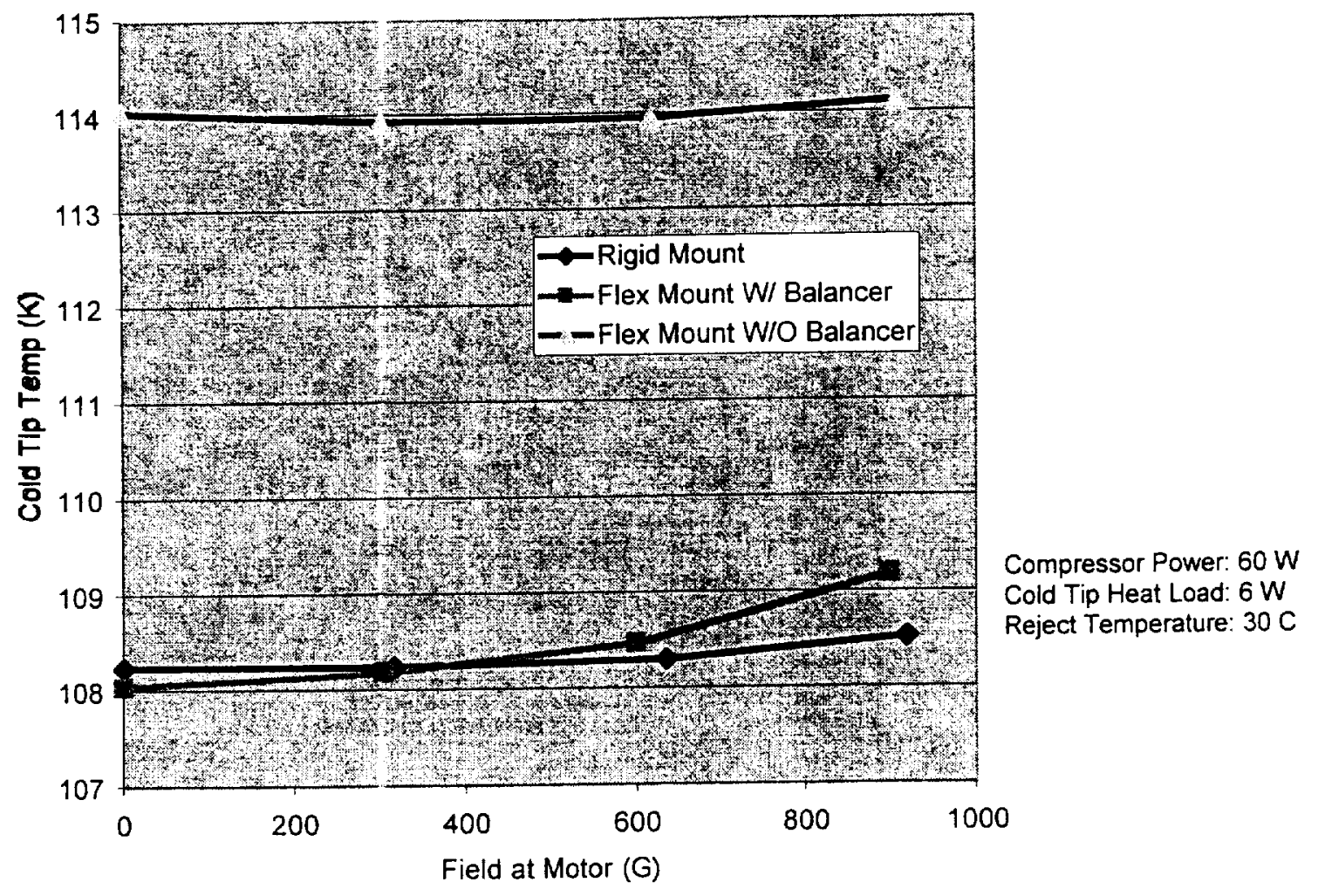

Figure 7: Effect of balancer on cryocooler performance in a magnetic field

with the balancer interacting with the magnetic field. When the cryocooler is rigidly mounted it does not lose energy in the mount. It is interesting to note that there is still a small performance degradation even when the balancer is removed from the cryocooler. This might indicate a loss of motor efficiency due to magnetic field. Although it is possible that the effect is due to rubbing of the piston, the repeatability of the phenomenon and absence of hysteresis suggests that this is not likely.

Additional tests will be conducted at the MIT cyclotron facility to understand the performance degradation at high fields. We will also measure the acceleration of the cryocooler to determine if the vibration forces are increased by the interaction with the magnetic field. Alternatives to the magnetic steel ring in the balancer will be tested, including a nonmagnetic stainless steel ring and a lanninated nonmagnetic stainless steel ring. In addition, forces on a sample cryocooler motor will be measured.

\section{ANALYSES}

The complex geometres encountered in this problem do not yield easily to simple calculations. The need to perform finite element analyses of the interactions between the applied magnetic field and the cryocuoler is being evaluated. We have done a very rough estimate trying to bound the eddy current heating in the cold finger and the compressor piston as they move through the magnetic field gradient. We have assumed that the maximum resultant field in both of the locations is coaligned with the cylindrical axis and that the magnetic field gradient equals the maximum value at both locations. The eddy current heating, $P$, in a cylinder of radius $R_{0}$ and thickness $L$ moving sirusoidally in a non-uniform magnetic field with gradient $d B / d x$ is given by:

$$
\mathrm{P}=(\mathrm{dB} / \mathrm{dt})^{2} \mathrm{~L} \pi \mathrm{R}_{0}^{4} / 8 \rho
$$

where $\rho$ is the electrical resistivity and $d B / d t$ is: 


$$
\mathrm{dB} / \mathrm{dt}=(\mathrm{dB} / \mathrm{dx})(\delta \omega \cos \omega \mathrm{t})
$$

where $2 \delta$ is the peak-to-peak stroke of the piston and $\omega=2 \pi f$ where $f$ is the operating frequency of the cryocooler. The maxinum eddy current heating is equal to:

$$
\mathrm{P}_{\max }=[2 \pi \mathrm{f} \delta(\mathrm{dB} / \mathrm{dx})]^{2} \mathrm{~L} \pi \mathrm{R}_{0}{ }^{4} / 8 \rho
$$

Similarly for a ring of thickness $L$ with outer radius $R_{0}$ and inner radius $R_{i}$ :

$$
P_{\max }=[2 \pi f \delta(d B / d x)]^{2} L \pi\left(R_{0}{ }^{4}-R_{i}{ }^{4}\right) / 8 \rho
$$

The maximum heating in the cold finger is approximately $1 \mathrm{~mW}$ and in the compressor is approximately $100 \mathrm{~mW}$. Thi: amount of heating is negligible for the cryocooler performance.

\section{CONCLUSION}

The Sunpower M87N cryocooler is capable of operating in a magnetic field as large as 1200 gauss perpendicular to the axis of the cryocooler. We are able to start and stop the cryocooler in a 1200 gauss field without difficult. A $1-4 \%$ thermal performance degradation has been seen at fields between 900 and 1200 gauss. Part of that degradation is attributed to an unbalancing of the passive balancer, resulting in additional energy loss in the flexible mounts. We are exploring the possibility of eliminating this effect, but still expect to see a very small degradation at high fields due to magnetic field effects on the motor..

The true test of operating the cryocooler in the AMS-02 magnetic field will only occur when the flight hardware is integrated and operated together. Any long-term degradation will not be known for certain until AMS-02 is on-orbit. The test program we have conducted has given us confidence that the short-term performance degradation will be negligible even up to 1.5 to 2 times the maximum expected field. Additional tests and analyses are planned to examine the question of long-term degradation that could lead to the failure of a cryocooler before the end of the mission.

\section{ACKNOWLEDGMENTS}

The authors wish to acknowledge the support of Dr. Henning Leidecker of NASA Goddard Space Flight Center in formulating approaches to the problem. We also wish to thank Dr. Ulrich Becker of MIT for access to the cyclotron facility and Messrs. Stephen Harrison and Steve Milward of Space Cryomagretics, Ltd. for calculations of the magnetic field parameters.

\section{REFERENCES}

1. Shirey, K.A., Banks, I.S., Breon, S.R., and Boyle, R.F., "Space Flight Qualification Program for the AMS-02 Commerical Cryccoolers," to be presented at the $12^{\text {th }}$ International Cryocooler Conference, Cambridge, MA, 2002.

2. Green, M.A. and Castles S, "Design Concepts for the Astromag Cryogenic System," Advances in Cryogenic Engineering, Vol. 33, Plenum Press, New York, 1988, pp. 631-637.

3. Kelley, R.L. et al., "The A stro-E High Resolution X-Ray Spectrometer," Proceedings of the SPIE No. 3765, Denver, CO, 1999.

4. Hoenig, M.O., "Design concepts for a mechanically refrigerated $13 \mathrm{~K}$ superconducting magnet system," IEEE Trans. On . Magnetics, Vol. MAG-19, May 1983.

5. Urata, M., et al., "Compac $17 \mathrm{~T}$ epoxy-impregnated magnet without bore tube," Cryogenics, Vol 31 , 1991 , pp. $570-574$. 
6. Laskaris, E.T., Ackermann, R., Dorri, B., Gross, D. Herd, K., and Minas, C., "ACryogen-Free Open Superconducting Magnet for Inte ventional MRI Applications," IEEE Transactions on Applied Superconductivity, Vol. 5, 1995, p. 163-168. 